\title{
RESECTION OF TUBERCULOUS STRICTURES OF THE MAIN BRONCHI IN THREE CASES \\ BY
}

\author{
J. W. JACKSON, P. H. JONES, AND T. HOLMES SELLORS \\ From the London Chest and Harefield Hospitals
}

(RECEIVED FOR PUBLICATION APRIL 28, 1955)

Until recent years the excision of an obstructive lesion of a bronchus has had to include the corresponding lung segment. In many cases this lung tissue is irretrievably damaged by bronchiectasis or infective changes and requires removal, but on occasions the lung is not diseased and merits preservation. The introduction of plastic surgery of the bronchus is one of the latest developments in thoracic surgery and has proved itself of considerable value in conservation of pulmonary tissue-a feature of great importance in the treatment of pulmonary tuberculosis.

Many surgeons have had experience of reconstructive operations of the bronchus after pulmonary resection, principally where a growth has extended along a main bronchus into the wall of the trachea or where an adenoma has invaded part of a bronchus which the surgeon had hoped to leave intact, but the records of success in the treatment of tuberculous strictures are infrequent. Direct closure of a defect made in a bronchus is a s.mple procedure and can be carried out effectively wherever sutures can be placed without narrowing other bronchi. The closure of a bronchial stump after lobectomy or pneumonectomy is the most common example. If sutures are likely to lead to narrowing, the defect can be closed by "patching" or covering, and here dermal grafts (Gebauer, 1950) have earned considerable popularity. The use of fascia lata and lung affords no problem so long as the defect is not so large that the graft is not supported and falls or is sucked into the bronchus or trachea and obstructs it. In such cases a framework of stainless steel or tantalum gauze gives support to the graft or the graft can be stiffened by steel threads passed through it.

Resection of a wedge of bronchus followed by direct suture has also been carried out on many occasions, but complete sleeve resection has been performed less frequently. Early attempts at bronchoplastic operations carried the risk of fistula formation with resulting pleural infection and non-expansion of lung-circumstances with which thoracic surgeons were only too familiar 10 or 15 years ago. The early successful sleeve resections were concerned with traumatic rupture of a main bronchus when the rigid wall of the tube was fractured, sometimes without loss of continuity of the mucosal lining. Direct end-to-end suture of a ruptured bronchus led to preservation of the lung in three instances in our experience, and recently attention was directed to pathological strictures beyond which there was complete or partial healthy lung tissue.

To put the problem simply: many patients with a stricture or benign tumour of a main stem bronchus were treated in the past by pneumonectomy because the risks of a local resection of the bronchus were too high. Now it seems possible to perform a sleeve resection in selected cases and yet preserve a lobe or the whole of the lung.

Tuberculosis is one of the most common causes of bronchial stricture, and the rôle played by endobronchitis in the course of the disease has been recognized as one of the most important pathological features. The healing of an endobronchial lesion, which is greatly helped by streptomycin, often leads to a fibrous stricture which may or may not be sufficiently severe to produce damage in the lung beyond. There are other methods of producing bronchostenosis, as for example by the external pressure of involved lymph glands or by ulceration of a caseous gland into the bronchial lumen. The site and severity of the stricture determine the fate of the lung, which may be left unaffected or may undergo atelectasis, bronchiectasis, abscess formation and retention, or pneumonitis. In some cases one lobe is unaffected while its neighbour is permanently damaged.

The present article is concerned with the treatment of strictures of the main bronchi in which the 
narrowing has not been so complete as to cause pathological changes in the whole lung, but has been restricted to damage and loss of function in one lobe only. One of the patients showed constriction in the left main bronchus with obliteration of the left upper lobe ; the other two were concerned with strictures in the right main bronchus in which the right upper lobe was completely destroyed. In all cases an upper lobectomy was performed and at the same time a ring-shaped length of the main bronchus was excised and continuity restored with an end-to-end junction. In this way a useful functioning lower lobe was preserved.

The problem of ensuring early and complete healing of a bronchial anastomosis is dependent on a variety of factors. The most important points are the production of an air-tight and accurate suture line between the divided ends, and early and complete re-expansion of lung so that no dead space persists. Antibiotics contribute to the control of infection, and in tuberculous patients they are of great value, as it is almost certain that some tuberculous tissue will have been incised during the course of the operation. The bronchial artery supply to the distal segment is inevitably interrupted by the division of the main stem bronchus, but this does not appear to jeopardize the vitality of the junction.

The technical points in sleeve resection include a clean upper cut above the tuberculous stricture and fashioning the lower cut obliquely so that the bronchus when rejoined is directed more horizontally and laterally than it would be normally. Non-absorbable sutures are used and two or three mattress stitches on the posterior border give good approximation and support to the rest of the anastomosis. The slightly everted edge can then be secured by fine additional stitches. It was not found necessary to use any flap or graft of tissue round the suture line, and in the cases reported there were no complications and to date there has been no recurrence of the stenosis at the site of the junction.

In general, resection of tuberculous strictures is more hazardous than the resection of simple strictures or of a simple growth within a bronchus, but a number of recent reports have been encouraging. Mathey and Maillard (1954) record three successful sleeve or ring resections for tuberculous stricture and Paulson and Shaw (1955) another three satisfactory examples. The field for this form of surgery is not extensive, but its ability to conserve healthy lung tissue in a number of patients constitutes a definite advance.

\section{CASE Reports}

CASE 1.-D. N., a woman aged 24 years, was diagnosed as having pulmonary tuberculosis at the age of 15 , when an abnormal shadow at the left hilum was noted and the sputum was positive for tubercle bacillus. After six months in hospital she was discharged home free of symptoms and remained well until May, 1953, when she developed persistent cough with $2 \mathrm{oz}$. of purulent sputum a day. The sputum was negative and the radiographic appearances had not changed. She was treated with penicillin and postural drainage, with marked improvement of symptoms. In November, 1953, the sputum was positive.

On admission to the London Chest Hospital in January, 1954, she had a cough with $1 \mathrm{oz}$. purulent sputum and some dyspnoea on exertion. She complained of a persistent wheeze in the chest, and this wheeze was clearly audible at the foot of the bed and made worse by lying on the left side. Sputum was positive, and the radiographs showed a calcified opacity projecting from the left hilum.

Bronchograms showed a stenosis of the left main bronchus beginning just below the carina and terminating below the orifice of the left upper lobe. There were bronchiectatic changes in the lingular and anterolateral segments of the upper lobe and the apicoposterior segments were not filled. The lower lobe was normal.

Bronchoscopy showed marked stenosis of the left main bronchus, beginning $1 \mathrm{~cm}$. below the level of the carina. The bronchus was uniformly narrowed to a lumen of $4 \mathrm{~mm}$. The mucosa was normal and the wall rigid.

A period of four months' chemotherapy (streptomycin, P.A.S., and isoniazid) was given with some general improvement.

Thoracotomy (May 10, 1954).-The patient was in the prone position and the pleural cavity was entered through the bed of the fifth rib. The left upper lobe was smaller than normal and nodular disease was palpab'e, mainly in the anterior segment. There were enlarged peri-bronchial lymph nodes, but no disease in the lower lobe. The upper lobe was dissected free and its bronchus divided after three arteries and the superior pulmonary vein had been secured. The lymph glands were removed from the main bronchus where they were very adherent, and the pulmonary $\sigma$ artery freed from the posterior aspect of the main bronchus. The whole length of the main bronchus $N$ from the carina to the lower lobe branches was $\omega$ cleared and the stenotic area resected. The sleeve removed was $1 \frac{1}{4}$ in. long and extended from the carina 0 close to the bronchus to the apex of the lower lobe. The two ends were brought together and an end-to- of end anastomosis carried out with interrupted mattress sutures of non-absorbable material. The junction was tested for air leak and the chest wall closed with drainage after satisfactory expansion of the lower lobe had been observed. 
The post-operative condition was satisfactory with good aeration of the left lower lobe, but on the second day partial atelectasis occurred; this was treated by bronchoscopic aspiration and resolved without incident. The subsequent course was uneventful, the remaining left lower lobe aerating satisfactorily. There was no evidence of wheeze.

Four months later bronchoscopy showed that the left main bronchus was patent. Its orifice was threequarters the normal size, but the junction permitted passage of the bronchoscope and full visualization of the lower lobe orifices. A suture projecting into the lumen on the medial wall was removed, and this was the only evidence of any abnormality.

The pathological report confirmed the presence of a strong fibrous stenosis beyond which there was bronchiectasis and widespread caseous tuberculosis. The bronchus at the point of section was oedematous but did not show giant cell systems. The general condition of the patient has remained completely satisfactory.

CASE 2.-F. G., a housewife aged 44 years, had been in ill health from 1942 following tonsillectomy. The following year she was married, but continued to work as a saleswoman. Two years later her health deteriorated, and in the autumn of 1946 a radiograph of the chest showed extensive infiltration of the right upper lobe. This improved during three months of bed rest. In August, 1948, there was a spread into the right mid-zone and progressive collapse of the right upper lobe was noted. In December, 1952, following removal of a uterine polyp, her general condition deteriorated and extensive disease in the apical segment of the right lower lobe was recorded. Sputum was positive for tubercle bacilli. A six months' course of chemotherapy produced some improvement.

On admission to Harefield Hospital at the beginning of 1954 she complained of dyspnoea on exertion and a wheeze which she stated had been present since 1945. This wheeze was easily audible from the end of the bed and there were rhonchi over the right chest. Her general condition was good; there was no cyanosis or clubbing.

In June, 1954, bronchography showed complete absence of any filling of the right upper lobe because of a stricture of the right main bronchus at the level of the upper lobe orifice. Bronchoscopy revealed a severe stricture of the right main bronchus close to the carina. The upper lobe orifice could not be seen. A course of chemotherapy was given for the next six weeks.

Thoracotomy (July 18, 1954).-With the patient in the face-down position, the pleural cavity was entered through the bed of the fifth rib. The right upper lobe was found shrunken, collapsed, and airless, firmly adherent in the greater fissure, with some calcified lesions felt in the apex of the lower lobe. The bronchus was dissected free with some difficulty, and a section of the bronchus from the carina to the level of the middle lobe orifice excised. The vessels to the upper lobe were secured and the upper lobe removed with the stenosed section of the main bronchus. Continuity was restored by an end-to-end anastomosis using interrupted mattress sutures. The lung re-expanded easily and the chest was closed with drainage.

Her post-operative convalescence was uneventful, there being normal breath sounds the day after the operation and no wheeze.

Bronchoscopy two months later showed a short right main bronchus which was not narrowed and with a direct view into the orifices of the middle and lower lobe branches. The line of suture could not be detected. A limited thoracoplasty was carried out, and the general condition of the patient has remained satisfactory.

The specimen showed the narrowed main bronchus with an upper lobe branch which was completely occluded. The lobe showed fibrotic changes of recent and healed tuberculous disease.

CASE 3.-M. H., a man aged 57 years, by occupation a civil servant, had pulmonary tuberculosis first diagnosed by mass radiography in June, 1945. Three years later the sputum was found to be positive, and he spent six months at home in bed. There was considerable improvement, but two years ago he developed bronchitis with cough and purulent sputum. The radiograph showed atelectasis of the right upper lobe ; sputum was positive. He was treated in a sanatorium by bed rest and chemotherapy.

Bronchoscopy in November, 1953, reported a stenosis of the right upper lobe orifice with scarring resulting from tuberculous endocarditis. In June, 1954, he had no symptoms, but the right upper lobe was atelectatic and small. In October, 1954, he was admitted to the London Chest Hospital. He had little sputum, which was negative, and the radiograph showed a collapsed right upper lobe with some infiltration and probably collapse of the middle lobe. Bronchoscopy showed stenosis of the right main bronchus, the opening being barely a quarter of the normal size and sited at the level of the carina; it was not possible to pass any instrument beyond this. A bronchogram confirmed the bronchoscopic findings and showed the absence of filling of the right upper lobe. A course of chemotherapy was started.

Thoracotomy (December 21, 1954).-With the patient in the face-down position, the pleural cavity was entered through the bed of the fifth rib. The right upper lobe was collapsed and shrunken and there was a palpable lesion in the middle lobe. The trachea and the right main bronchus were dissected free and the stricture excised by incision through the carina and on to the lateral wall of the trachea. Anaesthesia was maintained by a cuff tube guided down the left main bronchus. The bronchus below the stricture was then divided just above the middle lobe opening, and this section containing the stricture was removed with the upper lobe after the appropriate vessels had been divided. Continuity was restored by an anastomosis of the bronchus 
Fig. 1.-Case 1. Pre-operative radiograph showing calcified mass at the left hilum.

FIGs. 2 AND 3.-Case 1. Bronchograms showing stricture close to the trachea and no filling of the upper lote.

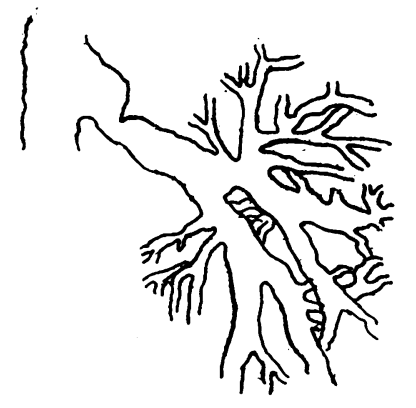

Fig. $2:$

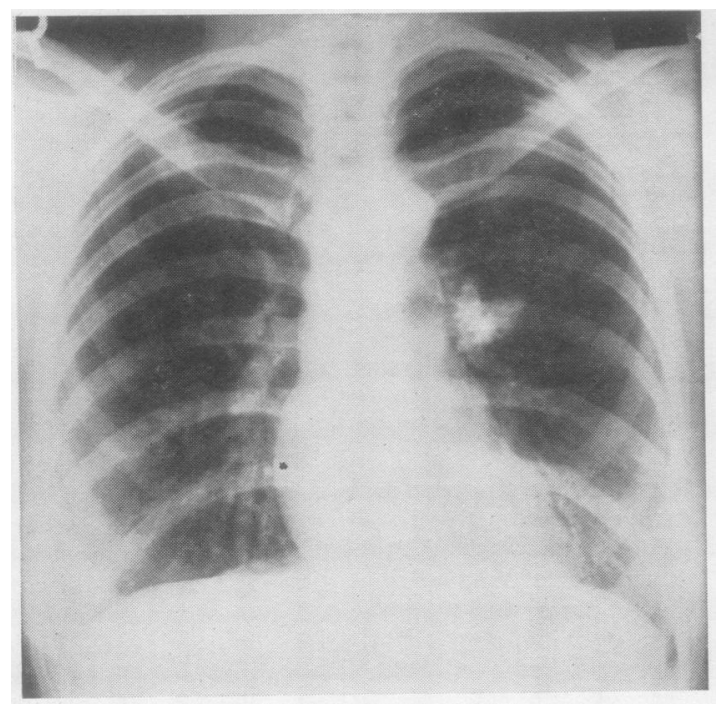

Fro. 1

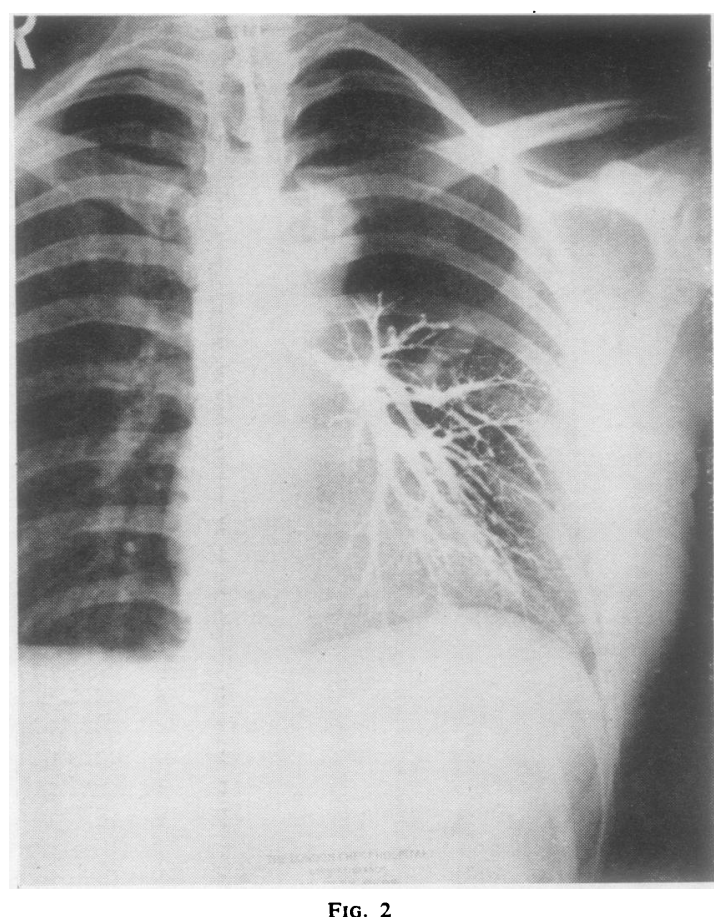

FIG. 2

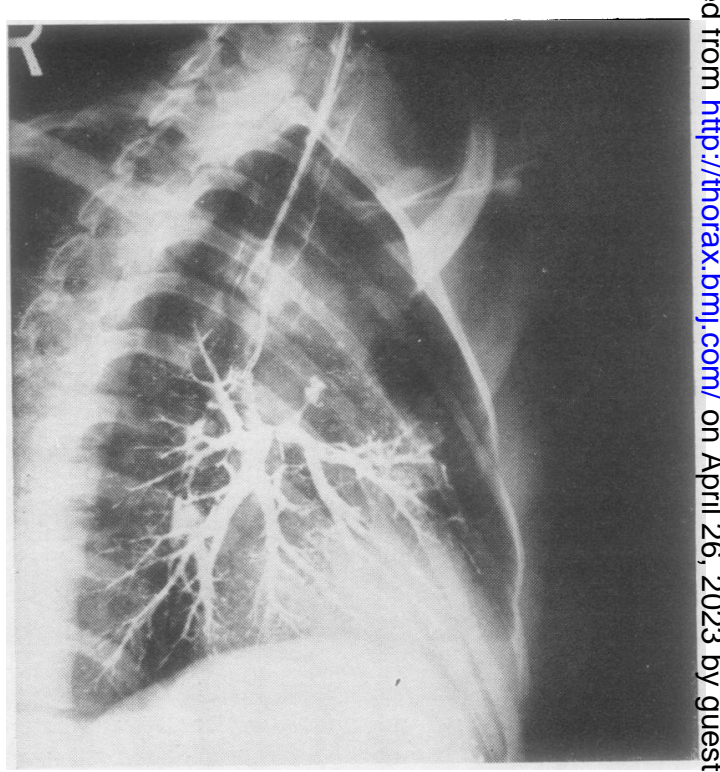

Fig. 3 


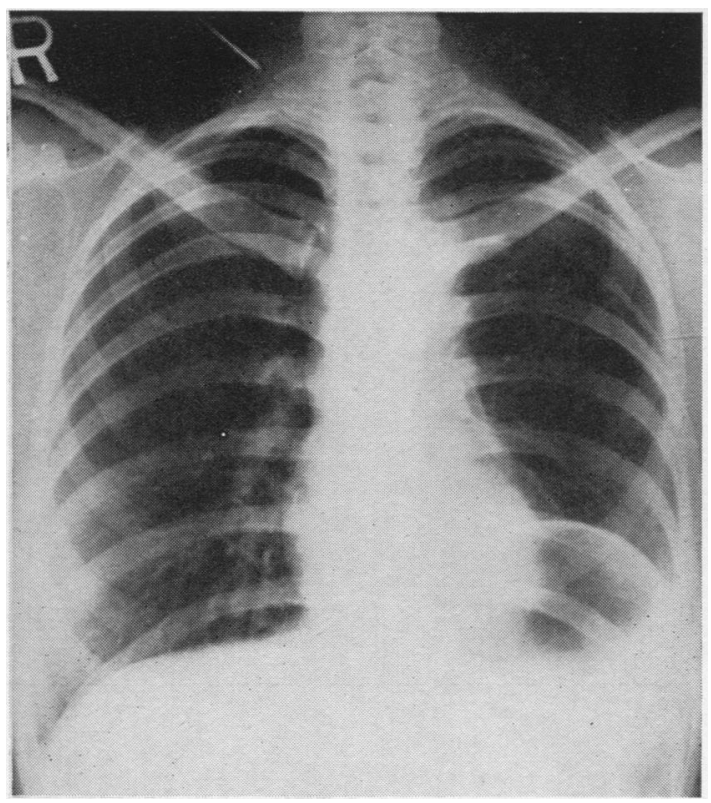

Figs. 4 AND 5.-Case 1. Post-operative radiographs. The outline of the left bronchus shows no narrowing.

Fig. 4

FIG. 6.-Case 1. Pre-operative radiograph showing complete collapse of the right upper lobe.

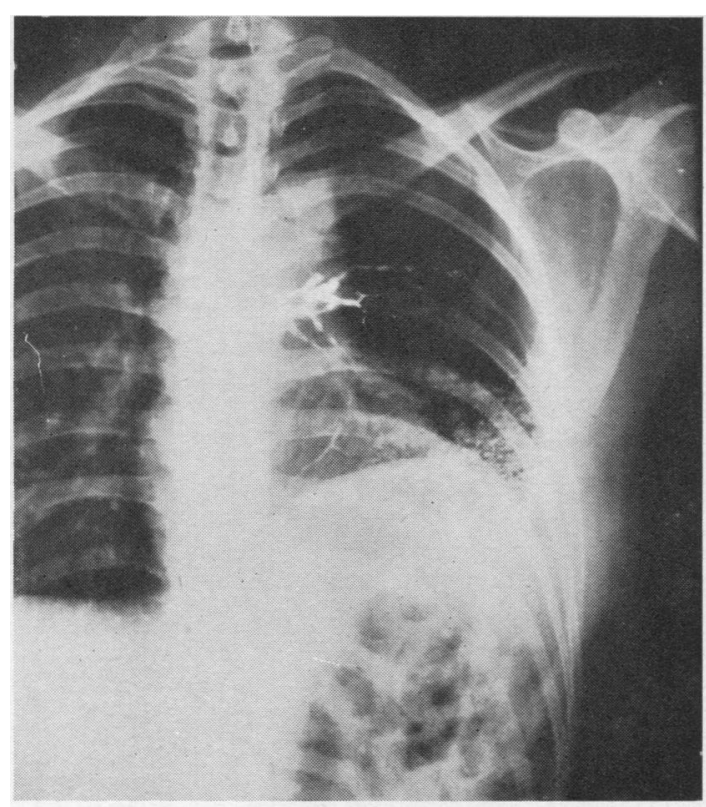

Fig. 5

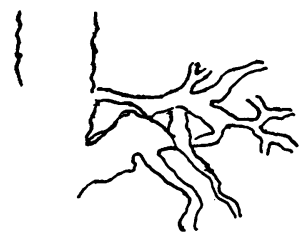

Fig. 5a

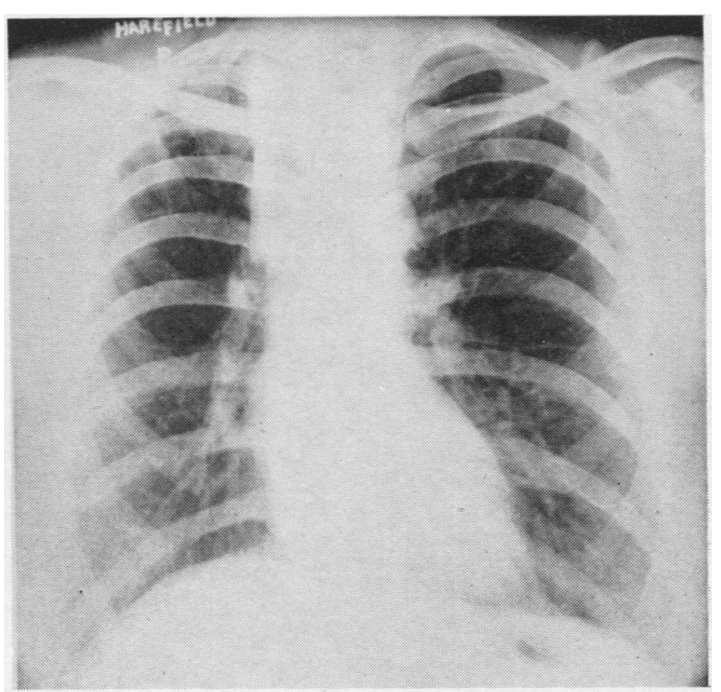

Fio. 6 


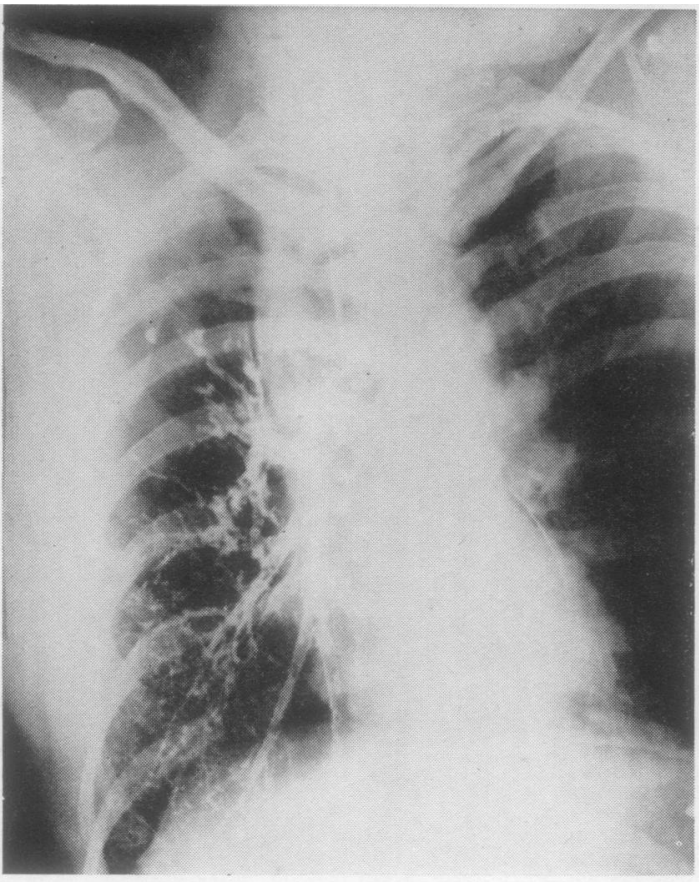

FIG. 7

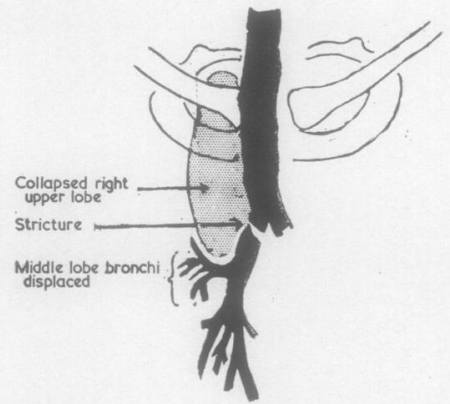

FIg. 7a

Figs. 7 and 8.-Case 2. Bronchograms showing stricture just beyond the tracheal bifurcation, non-filling of the upper lobe, and bronchiectasis of the middle lobe.

Fig. 9.-Case 2. Post-operative appearance. A small thoracoplasty has been performed in addition to the resection of the stricture and upper lobe.
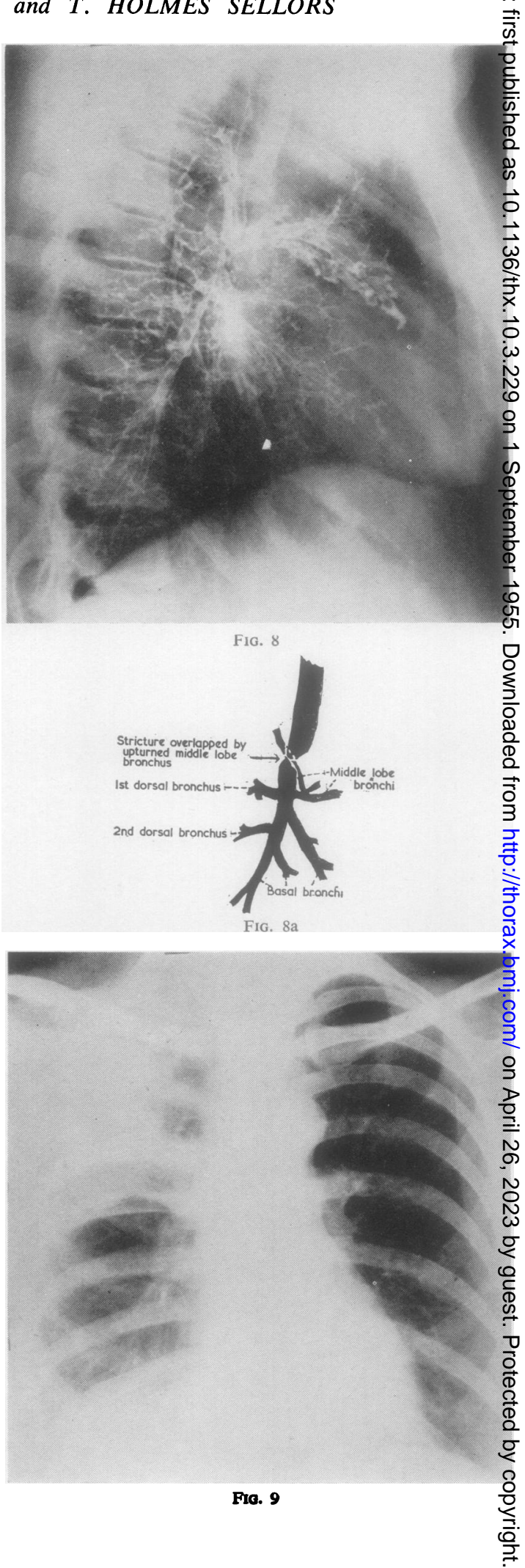


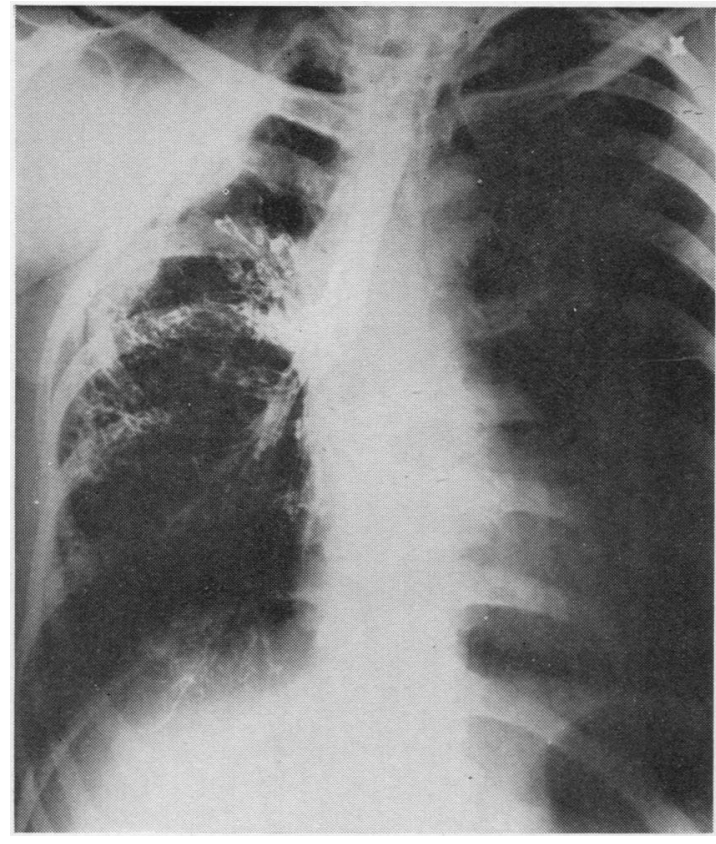

Fro. 10

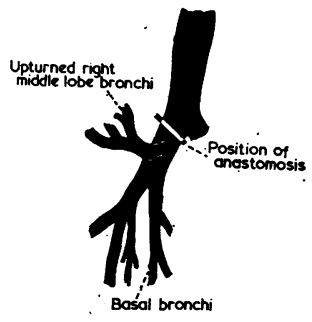

Fig. 10a

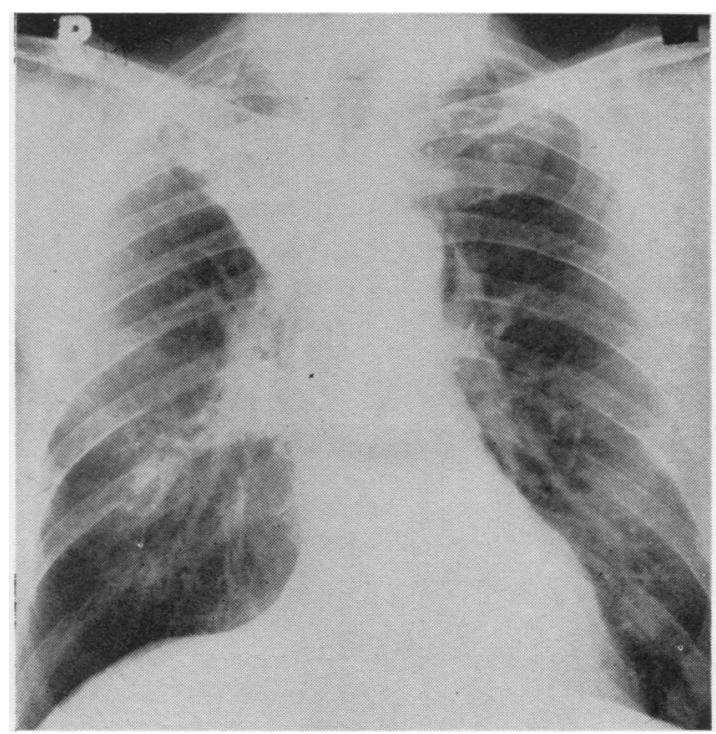

Fig. 12

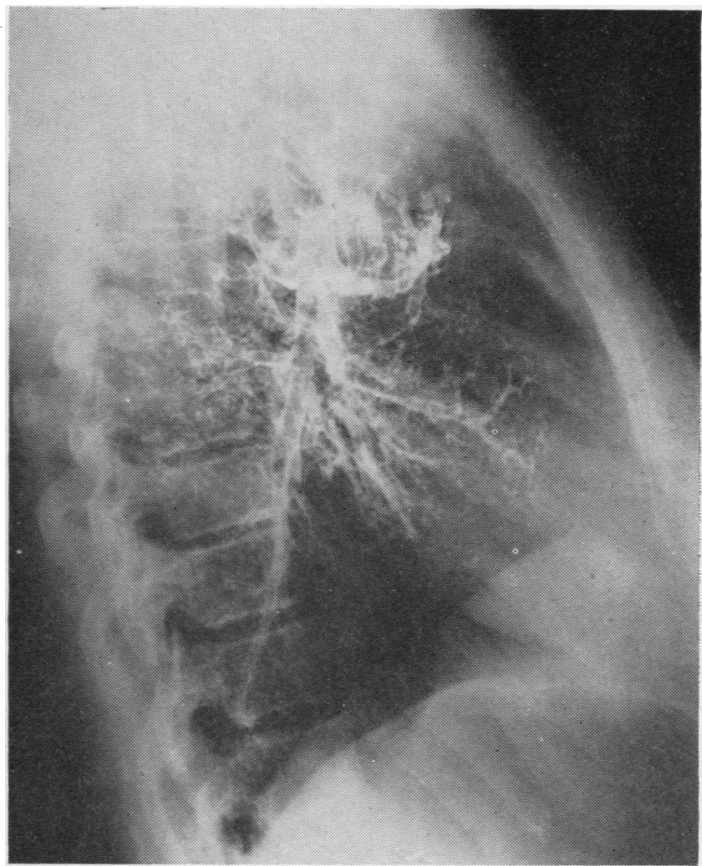

Fro. 11

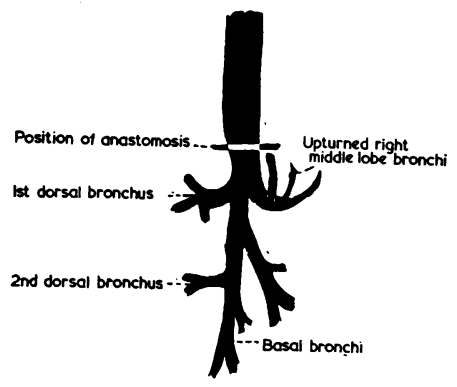

Fig. 11a

FIGs. 10 AND 11.-Case 2. Bronchograms showing absence of any stricture in the right main bronchus, which is considerably shortened.

FIG. 12.-Case 3. Pre-operative radiograph showing collapse of the right upper lobe. 


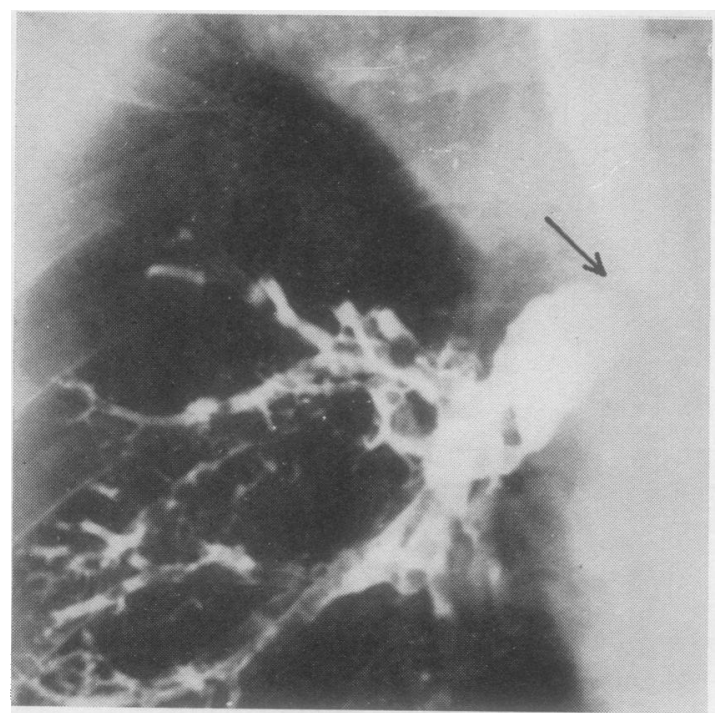

FIG. 13.-Case 3. Bronchogram showing stenosis where the right stem emerges from the trachea and no sign of an upper lobe bronchus.

direct on to the trachea, interrupted non-absorbable stitches being used. The suture line was reinforced by a flap of mediastinal pleura and the middle and lower lobes were easily expanded before the chest was closed with drainage. A modified thoracoplasty was performed to reduce space and to control the disease in the middle lobe.

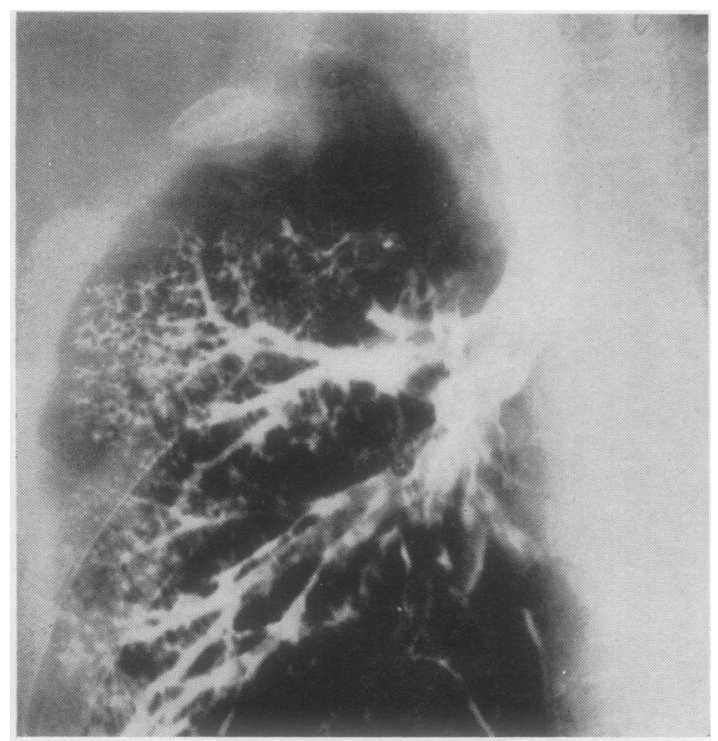

FIG. 15.-Case 3. Bronchogram showing no narrowing at the site of the tracheo-bronchial anastomosis.

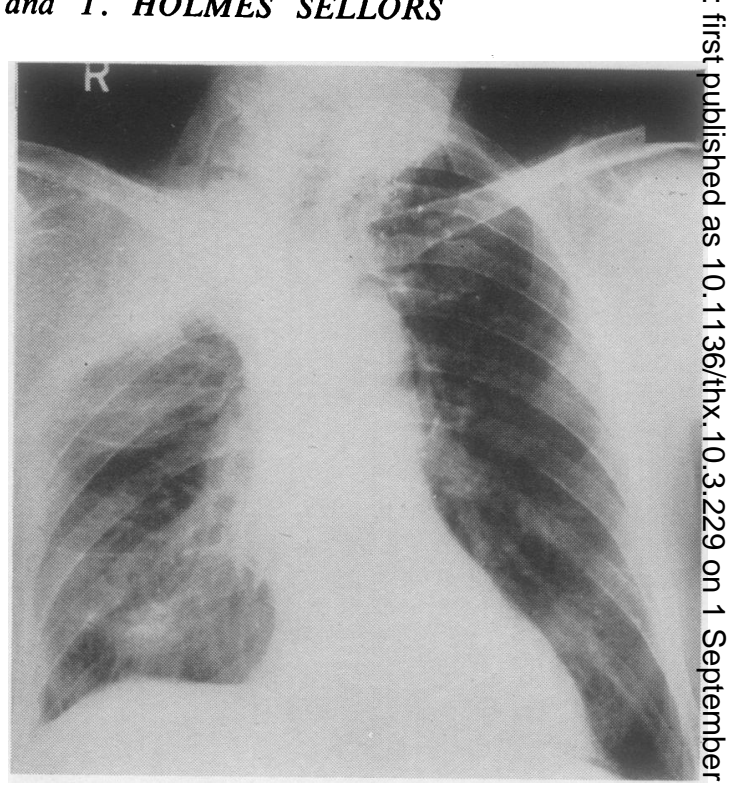

FIG. 14.-Case 3. Post-operative radiograph. A small thoracoplasty $\frac{1}{6}$ has been performed.

The post-operative course was uneventful; there was a minimal air leak, and the tubes were removeds three days after operation. Bronchoscopy six weeks after operation showed a normal calibre of then bronchus, and the only evidence of the anastomosis was a small irregular pit on the medial wall at the level of the carina. A bronchogram confirmed these $\vec{\sigma}$ findings. The patient's general condition has re- -3 mained satisfactory.

\section{SUMMARY}

Tuberculous strictures of the main bronchi may produce secondary changes involving a lobe or the whole of the lung. This depends on the site and severity of the narrowing. In some cases surgicaf. treatment involves the sacrifice of the whole lung in others excision of the stricture and restoration of continuity may allow a considerable part of the lung to be preserved.

Three cases are described in which a tubercu $\frac{7}{3}$. lous stricture had destroyed one lobe only. Thơ stricture and diseased lobe were excised and an end-to-end anastomosis performed. A successfub result was achieved in each case, the remainder of the lung being preserved.

\section{BIBLIOGRAPHY}

Belcher, J. R. (1950). Brit. J. Surg., 38, 121.

Carter, M. G., and Strieder, J. W. (1950). J. thorac. Surg., 20, 613. Gebauer, P. W. (1950). Ibid., 18, 604.

Gravel, J. A. (1954). Ibid., 27, 244.

Jackson, T. L., Lefkin, P., Tuttle, W., and Hampton, F. (1949)(্ণ) Ibid., 18, 630 .

Mathey, J., and Maillard, J. N. (1954). Sem. Hôp. Paris, 7, 1.

Paulson, D. L., and Shaw, R. R. (1955). J. thorac. Surg., 29, 238. 